\title{
¿Qué saben sobre el Tiempo Histórico los estudiantes de una escuela de enseñanza media para adultos de la ciudad de Santa Fe-Argentina?
}

\author{
Lucrecia Milagros Alvarez ${ }^{1}$
}

\begin{abstract}
RESUMEN
Este artículo presenta el análisis de un cuestionario semi-estructurado de elaboración propia, realizado a estudiantes de una escuela de enseñanza media para adultos de la ciudad de Santa Fe, Argentina. El mismo, forma parte del trabajo de campo de una futura tesis doctoral en el ámbito de la Didáctica de las Ciencias Sociales y de la Historia. El problema a investigar es el siguiente: ¿Cuáles son las representaciones sociales sobre el tiempo histórico que tienen los estudiantes jóvenes y adultos al comenzar el último año de su escolaridad, y al finalizar la enseñanza de una unidad didáctica?, y ¿De qué manera el desarrollo de una innovación contribuye a la mejora de la propia práctica de enseñanza? Con este trabajo se pretende contribuir a la vacancia de investigaciones que relacionen la enseñanza del tiempo histórico, las representaciones sociales de alumnos jóvenes y adultos acerca de este concepto, y la investigación sobre la propia práctica de la enseñanza.
\end{abstract}

PALABRAS CLAVES: Tiempo histórico. Representaciones sociales. Enseñanza de las Ciencias Sociales y la Historia. Escuela de enseñanza media para adultos. Investigación sobre la propia práctica.

O que os alunos de um ensino médio para adultos da cidade de Santa Fé-Argentina sabem sobre o Tempo Histórico?

\section{RESUMO}

\footnotetext{
${ }^{1}$ Profesor de Historia y Maestría en Didáctica Específica por la Universidad Nacional del Litoral - Facultad de Humanidades y Ciencias, Santa Fe, Argentina. Membro do Projeto CAI + D 2016: Práticas pedagógicas inovadoras em Geografia e História que promovam o pensamento crítico. https://orcid.org/0000-0001-6181-7872. lucreciamilagros@gmail.com.
} 
Este artigo apresenta a análise de um questionário semiestruturado de nossa própria elaboração, aplicado a alunos de uma escola secundária para adultos da cidade de Santa Fé, Argentina. Faz parte do trabalho de campo de uma futura tese de doutorado na área de Didática das Ciências Sociais e História. O problema a ser investigado é o seguinte: Quais as representações sociais sobre o tempo histórico que os alunos jovens e adultos têm no início do último ano de escolaridade e no final do ensino de uma unidade didática?, $\mathrm{E}$ de que forma $\mathrm{O}$ desenvolvimento de uma inovação contribui para a melhoria da prática docente? O objetivo deste trabalho é contribuir para a vaga de pesquisa que relaciona o ensino do tempo histórico, as representações sociais de jovens e adultos sobre esse conceito e a pesquisa sobre a própria prática docente.

Palavras-chave: Tempo histórico. Representações sociais. Ensino de Ciências Sociais e História. Ensino médio para adultos. Pesquisa na própria prática.

What do students of a middle school for adults in the city of Santa Fe-Argentina know about Historical Time?

\begin{abstract}
This article presents the analysis of a semi-structured questionnaire of our own elaboration, made to students of a secondary school for adults in the city of Santa Fe, Argentina. It is part of the field work of a future doctoral thesis in the field of Didactics of Social Sciences and History. The problem to be investigated is the following: What are the social representations about the historical time that young and adult students have at the beginning of the last year of their schooling, and at the end of the teaching of a didactic unit?, and in what way Does the development of an innovation contribute to the improvement of one's teaching practice? The aim of this work is to contribute to the research vacancy that relates the teaching of historical time, the social representations of young and adult students about this concept, and research on the teaching practice itself.
\end{abstract}

KEYWORDS: Historical time. Social representations. Teaching of Social Sciences and History. Middle school for adults. Research on own practice. 
$$
* * *
$$

Deconstruir el tiempo no quiere decir destruir los conocimientos históricos adquiridos, sino reinventar el tiempo en función de nuevas perspectivas para su aprendizaje. Deconstruir el tiempo histórico quiere decir poner en evidencia los convencionalismos que han generado determinadas visiones de la historia, presentar las contradicciones que estas visiones provocan y, a la vez, ofrecer nuevos enfoques de comprensión de la temporalidad humana. PAGÈS y SANTISTEBAN (1999, p. 195)
\end{abstract}

\title{
Introducción
}

Este artículo presenta aproximaciones iniciales del trabajo de campo de una futura tesis doctoral en el ámbito de la Didáctica de las Ciencias Sociales y de la Historia, realizado en una escuela de enseñanza media para adultos de la ciudad de Santa Fe, Argentina.

El problema a investigar es el siguiente: ¿Cuáles son las representaciones sociales sobre el tiempo histórico que tienen los estudiantes jóvenes y adultos al comenzar el último año de su escolaridad, y al finalizar la enseñanza de una unidad didáctica?, y ¿De qué manera el desarrollo de una innovación contribuye a la mejora de la propia práctica de enseñanza?

Como primer acercamiento al campo, hemos aplicado un cuestionario semi-estructurado de elaboración propia: en la primera pregunta los estudiantes debían seleccionar con cuáles de las siete frases que presentamos relacionaban el tiempo histórico. En una segunda, elegir entre cuatro definiciones del tiempo histórico cuál de ellas lo expresaba mejor y argumentar su respuesta.

Esta propuesta, es una continuidad de mi tesis de Maestría en Didácticas Específicas "La enseñanza de las categorías temporales en 
relación con la Historia Reciente. Análisis de prácticas de enseñanza de estudiantes avanzados de la carrera de Profesorado de Historia FHUC$U N L ” 2$, defendida en el año 2017, en la Universidad Nacional del Litoral.

En aquella oportunidad, la investigación realizada fue cualitativa, crítica e interpretativa y tomó la forma de un estudio de caso. La problemática inicial que motivó su elaboración, fue el desafío de enseñar el pasado cercano, sondeando específicamente las categorías temporales que los futuros profesores de Historia utilizaron en las prácticas finales de la carrera.

La confirmación del supuesto de la tesis realizada "Que el tiempo histórico no tiene un tratamiento específico o por lo menos no recibe la misma atención que períodos históricos concretos, porque existe un vacío de este contenido en las asignaturas disciplinares que lo enseñan de manera fragmentada o implicita”, durante la formación de los futuros profesores de Historia, nos invita nuevamente a profundizar la investigación sobre la temporalidad ${ }^{3}$. En esta oportunidad, en relación con los estudiantes jóvenes y adultos y sus representaciones sociales, a fin de poder realizar una propuesta de enseñanza innovadora para estas escuelas.

\section{Algunas precisiones metodológicas y conceptuales}

Como mencionábamos al inicio, esta investigación es una continuidad de mi tesis de maestría dado que profundiza cuestiones relacionadas con la enseñanza del tiempo histórico y se plantea como una investigación interpretativa, crítica, y un estudio de caso (Stake, 1999).

También, es una ruptura porque en esta nueva pesquisa doctoral se incorpora el concepto de representaciones sociales y cambia el sujeto de la investigación, los jóvenes y adultos que cursan el último año de escolaridad

\footnotetext{
2 Directora: Dra. Mariela Coudannes Aguirre. Co-director: Dr. Joan Pagès Blanch. En la tesis doctoral se continuaría con el mismo equipo de investigación, pero lamentablemente el maestro Joan ha fallecido en junio del corriente año. Este primer avance de la investigación, sería presentado en un artículo de escritura conjunta que nos quedó pendiente, espero estar a la altura de tamaño desafío: a él mi sentido e inacabable agradecimiento y cariño.

${ }^{3}$ En esta investigación los términos tiempo histórico y temporalidad se utilizan de manera intercambiable.
} 
en una de las divisiones de una escuela de enseñanza media para adultos (EEMPA) de la ciudad de Santa Fe.

Además, en relación a la metodología, se propone el análisis de la propia práctica de enseñanza, dado que la investigadora es simultáneamente la docente titular del curso. Nuestra investigación está orientada a la interpretación, para poder comprender situaciones complejas de enseñanza y aprendizaje, que en el aula de Ciencias Sociales se nos presentan como problemáticas.

En este trabajo sostenemos, además, un enfoque crítico buscando la comprensión atenta de la problemática que nos ocupa, con la finalidad de generar cambios y transformar la realidad educativa. Desde esta perspectiva, intentamos sondear las representaciones sociales que los estudiantes portan sobre el tiempo histórico y los procesos individuales, educativos, sociales, culturales que les dieron origen, antes y después de la enseñanza de una unidad didáctica.

Estamos convencidos de que las Ciencias Sociales, deben estar encaminadas a la construcción de un conocimiento social que contribuya a la creación de propuestas pedagógicas emancipatorias, que fortalezcan objetivos democráticos de los individuos organizados dentro de la sociedad (Imbernón, 2002).

Además, al tomar como punto de partida de nuestra investigación un problema concreto, nos transformamos en docentes-investigadores de nuestras propias prácticas. En palabras de Latorre (2003):

la enseñanza se concibe como una actividad investigadora y la investigación como una actividad autorreflexiva realizada por el profesorado con la finalidad de mejorar su práctica. La enseñanza deja de ser un fenómeno natural para constituirse en un fenómeno social y cultural, en una práctica social compleja, socialmente construida, e interpretada y realizada por el profesorado. (LATORRE, 2003, p. 9). 
En el marco de la investigación interpretativa y crítica que pretendemos realizar, utilizamos la metodología cualitativa a partir de la elaboración y aplicación de diversos instrumentos de investigación. En esta oportunidad, realizamos un cuestionario a los estudiantes de 5to año, con la finalidad de conocer las representaciones sociales que ellos tienen sobre el tiempo histórico al comenzar el cursado del último año de su trayecto formativo (Sanjurjo, 2005) en la EEMPA.

Después de analizar la información aportada por el mismo, con el objetivo de indagar en el origen de las representaciones sociales de los estudiantes, realizaremos entrevistas semi-estructuradas con algunos de ellos que sean representativos del grupo-clase. A partir de la obtención de estos resultados de la investigación, redefiniremos el programa de la asignatura para elaborar una unidad didáctica en la que se trabaje específicamente el tiempo histórico. Durante la enseñanza de la misma realizaremos grabaciones y observaciones participantes de las clases.

En la última etapa de la investigación, evaluaremos cambios y continuidades entre las representaciones sociales de la temporalidad al inicio de la escolaridad, y al finalizar la enseñanza de la unidad didáctica.

Simultáneamente, analizaremos el impacto de estos cambios en la propia práctica de enseñanza de la docente titular del curso.

El marco teórico de esta investigación, retoma el de la tesis de maestría realizada, en relación al concepto de tiempo histórico, que definimos desde el campo de la Didáctica de las Ciencias Sociales y de la Historia como:

- concepto estructurante de la disciplina (Benejam, 1999)

- metaconcepto polisémico (Pagès 1989; Pagès y Santisteban, 1999, 2008 y 2011)

- metacategoría (Pagès 1997a; Pagès y Santisteban, 2011)

- construcción social (Pagès y Santisteban, 1999; Santisteban 2007; Pagès 2014)

- construcción cultural (Pagès 1997a). 
Pagès $(1989 ;$ 1997a), diseña una estructura conceptual para la enseñanza del tiempo histórico y las categorías temporales que tomamos como referencia central en la tesis anterior, dado que una de las tensiones que para el autor atraviesan la escolaridad obligatoria, es que la temporalidad individual y social se construye por fuera de la escuela y además, no es un contenido central en los currículum de Historia y de Ciencias Sociales.

Complementariamente, Santisteban (2014) menciona las categorías temporales fundamentales a ser enseñadas en la escuela: el cambio y la continuidad, y la relación entre el pasado, el presente y el futuro.

Además, se incorporan en esta nueva investigación los últimos aportes que ha realizado la Universidad Autónoma de Barcelona, en Didáctica de las Ciencias Sociales y la Historia, provenientes del mismo grupo de investigación que tomamos como referente en la tesis anterior, relacionados con la educación para el futuro y los desafíos para la enseñanza y el aprendizaje de la temporalidad como: Anguera, 2012; 2013; Anguera y Santisteban, 2012; Santisteban y Anguera 2013 a y b; 2014; Llusá Serra y Santisteban, 2018; Santisteban, 2017; Pagès, 2019.

Para definir las representaciones sociales, tomamos como fuente principal el concepto de las investigadoras en Didáctica de la Geografía ZENOBI, "et al.", 2016, p.4, quienes se refieren a él como: “... una forma de conocimiento práctico, construido socialmente y compartido en el interior de los diferentes grupos. Se apoya en las experiencias de las personas, sirve para leer la realidad, actuar sobre ella y es guía en la vida cotidiana".

Esta categoría proviene de la psicología social, cuyo referente clásico es Moscovici (1986) y luego Jodelet (2011), pero desde el campo de la Didáctica de las Ciencias Sociales y la Historia, ha sido utilizada en múltiples investigaciones.

Algunas centradas en analizar las representaciones sociales y el tiempo histórico en la formación del profesorado, como: Hernández 
Cervantes y Pagès, 2014; Bravo Pemjean, Jara, y Llusá, 2017; Cartes Pinto, 2017 y 2020; Escribano, 2019; Escribano Muñoz y Gudín de la Lama, 2019; Escribano Muñoz, Pagès Blanch y Gudín de la Lama, 2019 y Escribano Muñoz y Pagès Blanch, 2019.

$\mathrm{Y}$ otras que investigan el concepto de representaciones sociales en diferentes escenarios educativos y temáticas como por ejemplo: el derecho, la justicia y la ley (Pagès y Oller, 2007); la participación democrática (Sant Obiols, Casas Ros y Pagès, 2011); y las crisis económicas (Olmos Vila y Pagès Blanch, 2017).

Queremos destacar, que existe una vacancia de investigaciones que relacionen el tiempo histórico y las representaciones sociales, en el contexto de la educación de adultos en Argentina, aporte que pretendemos realizar con esta investigación.

\section{Primeros avances en la investigación}

En la ciudad de Santa Fe, los estudiantes que por múltiples causas no han podido terminar la escuela secundaria común (orientada o técnica), tienen la posibilidad de continuarla o comenzarla en modalidad nocturna en las EEMPA. La formación se realiza en tres años, el $1^{\circ}$ año se cursa completo, $2^{\circ}$ y $3^{\circ}$ en el primer y segundo cuatrimestre (del segundo año de escolaridad) y lo mismo sucede con $4^{\circ}$ y $5^{\circ}$ año (en el tercer año).

La escuela en la que se realiza el trabajo de campo, está ubicada en una zona céntrica de la ciudad, a ella asisten estudiantes provenientes principalmente de los barrios populares. La mayoría de ellos son trabajadores y trabajadoras informales y amas de casa cuya franja etaria abarca entre los 18 y 45 años de edad; también hay una proporción relevante de jóvenes de 18 a 25 años que no trabajan ni estudian.

El grupo de estudiantes con el que llevamos a cabo la aplicación del cuestionario, corresponde a una de las divisiones que tiene la escuela para el último año de escolaridad. La proporción de mujeres y varones es 
similar, en un grupo-clase de 35 estudiantes en total que cursaron la materia en el segundo cuatrimestre de 2018.

Como parte del cuerpo docente de las EEMPA desde el año 2015, venimos constatando como problemática relevante que los estudiantes han llegado a la edad adulta, sin construir durante la escuela primaria y secundaria (en el caso de que hayan cursado algunos años en ella), una conceptualización sobre el tiempo histórico.

Por ello, consideramos que es fundamental conocer cuáles son las representaciones sociales sobre la temporalidad que tienen estos estudiantes al iniciar el cursado de la asignatura Ciencias Sociales ${ }^{4}$, dado que indagar su origen posibilitará la construcción de una propuesta de enseñanza innovadora que propicie su modificación y/o mejora.

De un total de 35 estudiantes matriculados en este curso, el cuestionario fue realizado por 21 , que asisten de manera regular a las clases de esta materia.

En la primera pregunta, debían responder marcando con una cruz, con qué se relacionaba para ellos el tiempo histórico, eligiendo entre las siguientes oraciones:

Fechas, años, décadas, siglos

La crisis del 2001 y sus consecuencias

El 25 de mayo de 1810

Lo que cambia y lo que continúa entre los gobiernos kirchneristas y el gobierno actual

El paso del siglo XX al siglo XXI

Lo que cambiará y continuará en la economía actual de nuestro país y el futuro por venir

La relación entre el pasado, el presente y el futuro

\footnotetext{
${ }^{4}$ Según la actualización de contenidos curriculares elaborada por el Ministerio de educación de la provincia (Circular 02/2019), se incluye en el área disciplinar de Ciencias Sociales a la Historia, la Geografía y la Formación ética y ciudadana. Para el caso de Historia de 5to año, se sugiere la enseñanza de temas vinculados con el contexto mundial, latinoamericano y argentino entre 1914 y la actualidad.
} 
La opción más elegida por los estudiantes, 15 del total, fue que el tiempo histórico se correspondía con la relación entre el pasado, el presente y el futuro. Como segunda opción, 4 estudiantes marcaron que la temporalidad hacía referencia a fechas, años, décadas, siglos. Cada una de las dos opciones siguientes -el tiempo histórico concernía al paso del siglo $\mathrm{XX}$ al siglo XXI; lo que cambiará y continuará en la economía actual de nuestro país y el futuro por venir- fueron seleccionadas por 1 estudiante respectivamente.

Ningún estudiante seleccionó que el tiempo histórico se relacionaba con: la crisis del 2001 y sus consecuencias; el 25 de mayo de 1810; y lo que cambia y lo que continua entre los gobiernos kirchneristas y el gobierno actual.

A partir de estas primeras respuestas de los alumnos, podemos suponer que conviven diferentes representaciones sociales de la temporalidad y que están presentes las categorías temporales que Santisteban (2014) mencionaba como centrales para ser enseñadas: cambios, continuidades, pasado, presente y futuro. En menor medida, aparecería también una representación del tiempo histórico asociada al positivismo, que lo vincularía con la cronología (fechas, años, décadas, siglos).

Nos interrogamos acerca de por qué los estudiantes no seleccionaron entre sus preferencias acontecimientos históricos relevantes para la historia de nuestro país, como el 25 de mayo de 1810, efeméride central del calendario escolar al recordarse el Primer gobierno patrio; o procesos más recientes como la crisis del 2001 o los gobiernos kirchneristas (2003-2015).

Podríamos inferir a partir de estas respuestas iniciales, que los estudiantes jóvenes y adultos que comienzan el 5to año en la EEMPA, tienen ciertas representaciones sociales sobre el tiempo histórico, y que un $25 \%$ lo relaciona con la cronología. 
Sin embargo, introducimos tensiones a fin de profundizar el análisis, dado que la mayoría de estos estudiantes habían cursado con la docente titular durante el primer cuatrimestre del año 2018, 4to año en la materia Ciencias Sociales. Por lo tanto, tenían cierta experiencia del trabajo con la temporalidad, que puede haber influido en que las respuestas mayoritarias hayan sido que el tiempo histórico se relaciona con el pasado, el presente y el futuro, por haber sido trabajado en diversas actividades de clases y en la explicación de la docente.

A comienzos del cursado de 4to año en la EEMPA, se realiza una actividad diagnóstico, a partir de la cual se elabora la planificación cuatrimestral de la materia. En la misma, se incluyó una pregunta sobre que era para ellos el tiempo histórico.

Los estudiantes respondieron que la temporalidad se relacionaba con: las fechas, el pasado, la duración, acontecimientos puntuales, un período durante el que se realiza una acción o se determina un acontecimiento, la medida de vida, un concepto que indica cuándo algo sucedió.

Es de destacar, que en aquella ocasión, la mayoría de los estudiantes no respondió o expresaba de forma oral no poder definir o "no haber dado ese concepto" en la escuela primaria y secundaria.

Complejizamos entonces nuestro punto de partida, porque en esta actividad de inicio escolar, no predomina una representación social de la temporalidad vinculada a la relación entre pasado, presente y futuro (elegida por la mayoría de los estudiantes en el cuestionario); sino que por el contrario, identificamos una mayoritaria que define al tiempo histórico asociándolo con la cronología, fechas, períodos.

Estas dificultades que advierten las investigaciones actuales en Didáctica de las Ciencias Sociales y de la Historia, pueden explicarse por múltiples razones:

1) la multiplicidad de significados que tiene el tiempo histórico, dificulta la comprensión del mismo (Pagès y Santisteban, 2010). 
2) la aceleración del tiempo por la rapidez en que se producen los cambios, y el valor que han adquirido los acontecimientos de corta duración en la actualidad, genera como problema la pérdida de la coherencia temporal: que las personas tengan dificultades para una comprensión real de la experiencia en la percepción del tiempo (Pagès, 1997a).

3) la concepción del tiempo histórico que ha sido predominante, y que persiste en la escuela es la positivista, presentando una visión sesgada, simple, lineal, uniforme, objetiva, acumulativa y determinista de la realidad, de la temporalidad y de la historia (Pagès 1997a; Pagès y Santisteban 1999 y 2010). En este marco influenciado por la pervivencia del positivismo, el tiempo histórico no es un objeto específico de enseñanza ni de aprendizaje.

4) la enseñanza y el aprendizaje de una estructura conceptual del tiempo histórico, no se desarrolla de manera explícita durante la formación del profesorado (Santisteban, 2000), porque no existen propuestas que guíen la misma. Supuesto que hemos confirmado en la tesis de maestría antes mencionada, para el caso de las asignaturas disciplinares del Profesorado de Historia de la Universidad Nacional del Litoral (Alvarez, 2017).

Podríamos suponer, que esta formación inicial endeble, impacta en la enseñanza que estos profesores llevarán a cabo en distintos niveles y modalidades del sistema educativo, una vez alcanzada su titulación de grado; lo que tendría influencias directas en el aprendizaje de los estudiantes.

En la segunda pregunta del cuestionario, los alumnos debían seleccionar entre cuatro citas reelaboradas por la docente-investigadora acerca del tiempo histórico, cuál era la definición más adecuada, y explicar el porqué de su elección. Debían elegir entre las siguientes: 
1) Una de las formas de ordenar los hechos de los más antiguos hasta la actualidad es la cronología. En una línea de tiempo se pueden colocar años, décadas, siglos y procesos históricos. ${ }^{5}$

2) Todas las historias, de la vida y del mundo, pueden presentarse como:

Acontecimientos, por ejemplo una batalla, un discurso, un encuentro entre presidentes de dos países.

Coyunturas, como una crisis económica que puede durar cinco, diez, treinta y hasta cincuenta años.

Procesos, tiempos más largos como por ejemplo lo que cambia y lo que continúa entre el siglo XIX y XX.6

3) Para entender qué es el tiempo histórico es útil saber lo que cambia y lo que continúa $y$, las relaciones que pueden establecerse entre el pasado el presente y el futuro.

Si pensamos en un reloj de arena, lo que ha caido es el pasado, lo que resta por caer es el futuro, y el paso por el que la arena cae de un lado a otro, es el presente. $^{7}$

4) Para construir una mejor sociedad con bienestar y desarrollo, es importante tener información sobre el mundo y lo que nos rodea, para poder imaginar distintos futuros. Desarrollar una visión crítica y responsable del hoy y de los futuros por venir. ${ }^{8}$

La definición uno, tres y cuatro fueron seleccionadas por 6 estudiantes respectivamente; la conceptualización dos, por 2 estudiantes. Sólo 1 estudiante manifestó no haber comprendido la pregunta, dado que eligió dos definiciones de la temporalidad (dos y tres). Por último, 3 estudiantes del total no lograron justificar el porqué de su elección.

Podemos suponer que en las respuestas dadas a esta segunda pregunta del cuestionario, se acentúa aún más lo que constatábamos en la primera, dado que conviven múltiples significados del tiempo histórico:

a) Una definición que lo relaciona con la cronología (número uno);

\footnotetext{
${ }^{5}$ Reelaboración propia de RIZZI, A.; “et al”. Una historia para pensar. La Argentina en el largo siglo XIX. Buenos Aires: Ed. Kapeluz, 2009, p. 14.

${ }^{6}$ Reelaboración propia de BRAUDEL, F. La Historia y las Ciencias Sociales. Madrid: Alianza Editorial, 1968, 1970, p. 27 y 53.

${ }^{7}$ Reelaboración propia de SANTISTEBAN, A. Tiempo al tiempo en la escuela: una mirada transversal. Revista Perspectiva Escolar - Monografías. Enseñanza de las Ciencias Sociales, No 1, p. 43-48, 2014, p. 6.

${ }^{8}$ Reelaboración propia de ANGUERA CERAROLS, C. Una investigación sobre cómo enseñar el futuro en la educación secundaria. Revista Enseñanza de las Ciencias Sociales. Revista de Investigación. Vol.12, p. 27-35, 2013, p. 30.
} 
b) Otra con los cambios, las continuidades y la relación entre pasado, presente y futuro (número tres);

c) Una que apela a la importancia de la enseñanza del y para el futuro (número cuatro);

d) En menor medida la definición de tiempo corto, medio y largo (número dos).

Además de seleccionar cuál era para ellos la definición más adecuada, debían justificar su elección. Incorporamos a continuación algunas de sus argumentaciones en las que conviven representaciones sociales que:

- Destacan la importancia de aprender sobre el tiempo histórico y la historia para no cometer los mismos errores del pasado y construir un mejor futuro: "Es necesario tener este conocimiento para evitar aquellos errores que marcaron la historia de forma negativa $y$ encontrar gente más capacitada en estos ámbitos para tomar mejores decisiones económicas y sociales para construir un mejor futuro donde el éxito sea igualitario” (E99)

- Expresan la relevancia de relacionar el presente y el futuro, y la importancia de tomar consciencia de estas relaciones para crear una sociedad mejor: "Porque para estar bien en la sociedad necesitamos estar más unidos y desarrollar un mundo mejor, un futuro dónde no haya más pobreza, delincuencia y egoísmo”(E20)

- Relacionan el pasado, el presente y el futuro: "Porque ayuda a analizar entre lo que ya pasó, lo que está pasando y cómo podemos mejorar a futuro" (E4)

- Vinculan al tiempo histórico con la cronología: "Los hechos históricos deben marcarse en una línea de tiempo, dándoles fechas y nombres para poder seguir un orden cronológico: para poder

\footnotetext{
${ }^{9}$ E9: Estudiante número 9.
} 
ubicarnos en tiempo y espacio en cada fecha en dónde se produjo un hecho importante que marcó nuestra historia” (E15)

\section{Las primeras conclusiones}

En el análisis que presentamos en este artículo realizamos un primer avance del trabajo de campo de una futura tesis doctoral, en relación con las representaciones sociales de estudiantes de 5to año de una EEMPA de la ciudad de Santa Fe.

Consideramos que a partir de las respuestas a las dos preguntas del cuestionario, podemos inferir que la mayoría de los estudiantes jóvenes y adultos han logrado:

a) Seleccionar que es el tiempo histórico para ellos, de una lista de opciones posibles.

b) Elegir y dar fundamentos acerca de diferentes definiciones de la temporalidad.

También, a partir de sus respuestas, hemos identificado tensiones en las representaciones sociales múltiples y variadas que los estudiantes tienen de la temporalidad, que nos llevan a interrogarnos:

- ¿Qué conceptualizaciones del tiempo histórico están presente realmente en la selección y argumentación realizada por los estudiantes?

- ¿Qué otras categorías temporales mencionarían como centrales dentro del metaconcepto tiempo histórico, además de las incluidas en el cuestionario? (cambios, continuidades, pasado, presente y futuro)

- ¿Cuál es el origen de esas representaciones de la temporalidad: sus realidades familiares, laborales, barriales, comunitarias, sus trayectorias escolares, la información que circula en los medios de comunicación (TV, radio, redes sociales)? 
- ¿Qué historia le han enseñado y han aprendido durante su escolaridad obligatoria, y cuál es su influencia en la construcción de sus representaciones sociales del tiempo histórico?

- ¿Qué materiales o recursos didácticos han utilizado los docentes en la primaria y secundaria, para que estos estudiantes hayan construido estas representaciones?

- ¿Qué cambios son necesarios introducir en la enseñanza de los contenidos curriculares de esta materia, fundamentalmente en Historia, para que sea significativa para estudiantes jóvenes y adultos del siglo XXI?

La aplicación de este instrumento de investigación nos ha dado un panorama inicial de las representaciones de la temporalidad que los estudiantes de esta escuela tienen al inicio del último año de escolaridad obligatoria.

Esta primera aproximación al campo, otorga herramientas potentes para continuar con la investigación, identificando estudiantes representativos del grupo-clase a los que realizaremos entrevistas semiestructuradas a fin de sondear el origen de estas representaciones sociales. Para luego, realizar la revisión de los contenidos de la planificación cuatrimestral, y el posterior diseño de una secuencia didáctica innovadora, en la que se trabaje de manera específica la temporalidad.

Luego de llevar a cabo la enseñanza de la secuencia, se podrán evaluar los cambios y continuidades entre las representaciones sociales del tiempo histórico al inicio y al final de este trabajo de campo; y simultáneamente el impacto de esta investigación en la propia práctica de la enseñanza.

\section{Referencias}

Actualización de contenidos curriculares de la Dirección Provincial de Educación Permanente de Jóvenes y Adultos. Ministerio de Educación de la Provincia de Santa Fe (Circular 02/2019). 
ALVAREZ, L. La enseñanza de las categorias temporales en relación con la Historia Reciente. Análisis de prácticas de enseñanza de estudiantes avanzados de la carrera de Profesorado de Historia, FHUC-UNL. 2017. 214 f. Tesis (Maestría en Didácticas Específicas) - Universidad Nacional del Litoral, Santa Fe.

ANGUERA, C. (2012). El concepto de futuro en la enseñanza de las ciencias sociales. Estudios de caso en la educación secundária.2012. 429 f. Tesis (Doctorado en Didáctica de las Ciencias Sociales) - Universidad Autónoma de Barcelona, Barcelona.

ANGUERA CERAROLS, C. Una investigación sobre cómo enseñar el futuro en la educación secundaria. Revista Enseñanza de las Ciencias Sociales. Revista de Investigación. Vol.12, p. 27-35, 2013.

ANGUERA, C.; SANTISTEBAN, A. El concepto de futuro en la enseñanza de las Ciencias Sociales y su influencia en la participación democrática. En: DE ALBA FERNÁNDEZ, N.; GARCÍA PÉREZ, F. Y SANTISTEBAN FERNÁNDEZ, A. (eds.) Educar para la participación ciudadana en la enseñanza de las Ciencias Sociales, Vol. 1, España: AUDCS y Díada Editora, S. L., 2012, pp. 391-400.

BENEJAM, P. La oportunidad de identificar conceptos clave que guíen la propuesta curricular de ciencias sociales. Revista ÍBER Didáctica de las Ciencias Sociales, Geografía e Historia No 21, p. 13-22, 1999.

BRAUDEL, F. La Historia y las Ciencias Sociales, Madrid: Alianza Editorial, 1968, 1970.

BRAVO PEMJEAN, L.; JARA, M. A.; LLUSÁ, J. La concepción del tiempo histórico en futuros docentes de Argentina, Chile y España. Pasado Abierto. Revista del CEHHis (6), p. 135-155, 2017.

CARTES PINTO, D. La enseñanza de la periodización en la formación inicial docente en la Universidad de la Frontera de Temuco, Chile. En: MARTÍNEZ, R.; GARCÍA- MORÍS, R.; GARCÍA, C. (edit.) Investigación en didáctica de las ciencias sociales. Retos, preguntas y líneas de investigación. Córdoba: Universidad de Córdoba/ AUPDCS, 2017, p. 52-61.

CARTES PINTO, D. La periodización y la conciencia histórica en la formación del profesorado. Revista REIDICS. Revista de Investigación en Didáctica de las Ciencias Sociales, Nº 6, Universidad de Extremadura: AUPDCS, p. 6-23, 2020.

ESCRIBANO MUÑOZ, C. Enseñar a enseñar el tiempo histórico. ¿Qué saben y qué aprenden los futuros docentes de secundaria? 2019. $300 \mathrm{f}$. Tesis. (Doctorado) Universidad Internacional de la Rioja, Madrid.

ESCRIBANO MUÑOZ, C.; GUDÍN DE LA LAMA, E. Comprender el sentido del tiempo histórico para enseñar la historia: una propuesta de investigación. En: BALLBÉ, M. GONZALEZ-MONFORT, N.; SANTISTEBAN, A. (eds.) Quin professorat, quina ciutadania, quin futur? Els reptes de l'ensenyament de les 
ciències socials, la geografia i la historia. Barcelona: GREDICS (Grup de Recerca en Didàctica de les Ciències Socials). Universitat Autònoma de Barcelona, 2019, p. 259-267.

ESCRIBANO MUÑOZ, C., PAGÈS BLANCH, J. (2019) Representaciones sociales de futuros docentes de historia sobre el tiempo y la conciencia histórica. Reseñas de Enseñanza de la Historia, № 17, Córdoba: Pueblo de la Toma, p. 17-35, 2019.

ESCRIBANO MUÑOZ, C.; PAGÈS BLANCH, J.; GUDÍN DE LA LAMA, E. Representaciones sociales de los estudiantes del máster de profesor de secundaria (geografía e historia) sobre la enseñanza y aprendizaje del tiempo histórico. Primera aproximación desde la teoría fundamentada. En: HORTAS, M.; DIAS, A.; DE ALBA, N. Enseñar y aprender didáctica de las Ciencias Sociales: la formación del profesorado desde una perspectiva sociocrítica. Lisboa: Ediciones Escola Superior de educação, Instituto Politécnico de Lisboa/AUPDCS, 2019, p. 335-345.

HERNÁNDEZ CERVANTES, L.; PAGÈS, J. ¿Qué es el tiempo?, ¿para qué y cómo se enseña? Las representaciones sociales de seis estudiantes de profesora en educación infantil sobre el tiempo histórico y su enseñanza. Reseñas de Enseñanza de la Historia, No 12, Córdoba: Pueblo de la Toma, p. 9-29, 2014.

IMBERNÓN, F. La investigación educativa como herramienta de formación del profesorado. Barcelona: Editorial Graó, 2002.

JODELET, D. Aportes del enfoque de las representaciones sociales al campo de la educación. Espacios en blanco. Revista de Educación vol. 21, p. 133-154, Junio, 2011.

LATORRE, A. La investigación-acción. Conocer y cambiar la práctica educativa. Barcelona: Ed. Graó, 2003.

LLUSÁ SERRA, J.; SANTISTEBAN, A. La construcción del tiempo histórico. En: JARA, M. A.; SANTISTEBAN, A. (coords.) Contribuciones de Joan Pagès al desarrollo de la didáctica de las ciencias sociales, la historia y la geografía en Iberoamérica. Cipoletti: UNCO/UAB, 2018, p. 43-53.

MOSCOVICI, S. Psicología Social II. Barcelona: Editorial Paidós, 1968.

OLMOS VILA, R.; PAGÈS BLANCH, J. Cambios y continuidades en las representaciones sociales de un mismo grupo de chicos y chicas sobre las crisis económicas. En: MARTÍNEZ, R. GARCÍA- MORÍS, R.; GARCÍA, C. (edit.) Investigación en didáctica de las ciencias sociales. Retos, preguntas y líneas de investigación. Córdoba: Universidad de Córdoba/ AUPDCS, 2017, p. 720-729.

PAGÈS, J. Aproximación a un currículum sobre el tiempo histórico. En: RODRÍGUEZ, J. (ed.) Enseñar historia. Nuevas propuestas. Barcelona: Laia/Cuadernos de Pedagogía, 1989, p. 107-138.

PAGÈS, J. (1997a) El tiempo histórico. En: BENEJAM, P.; PAGÈS, J. (coord.) Enseñar y aprender Ciencias Sociales, Geografía e Historia en la Educación 
Secundaria. Barcelona: Horsori, ICE-Universidad de Barcelona, 1997a, p. 189208.

PAGÈS, J. Conciencia y tiempo histórico. Revista Perspectiva Escolar Monografías. Enseñanza de las Ciencias Sociales, N 1, p. 35-40, 2014.

PAGÈS, J. Enseñar historia, educar la temporalidad, formar para el futuro. $E l$ Futuro del Pasado, 10, p. 19-56, 2019.

PAGÈS, J.; OLLER, M. Las representaciones sociales del derecho, la justicia y la ley e un grupo de adolescentes catalanes de $4^{\circ}$ de ESO. Enseñanza de las Ciencias Sociales, (6) p. 3-17, 2007.

PAGÈS, J., SANTISTEBAN, A. La enseñanza del tiempo histórico: Una propuesta para superar viejos problemas. En: PAGÈS, J.; SANTISTEBAN, A. Un currículum de Ciencias Sociales para el siglo XXI. Qué contenidos y para qué. Sevilla: Díada, 1999, p. 187-207.

PAGÈS, J., SANTISTEBAN, A. Cambios y continuidades: aprender la temporalidad histórica. En: JARA, M. A. (coord.). Enseñanza de la Historia. Debates y Propuestas (pp.). Neuquén: EDUCO - Editorial de la Universidad Nacional del Comahue, 2008, p. 95-127.

PAGÈS, J.; SANTISTEBAN, A. La enseñanza y el aprendizaje del tiempo histórico en la educación primaria. Cad. CEDES Campinas, vol. 30, nº 82, p. 281309, 2010.

PAGÈS, J., SANTISTEBAN, A. Enseñar y aprender el tiempo histórico. En: SANTISTEBAN, A.; PAGÈS, J. Didáctica del Conocimiento del Medio Social y Cultural en la Educación Primaria. Ciencias Sociales para comprender, pensar y actuar. España: Síntesis, 2011, p. 229-247.

RIZZI, A.; RAITER, B.; BROWQRNIK, G.; MANTIÑAN, C.; PINEAU, N. Una historia para pensar. La Argentina en el largo siglo XIX. Buenos Aires: Ed. Kapeluz, 2009.

SANJURJO, L. La formación práctica de los docentes. Reflexión y acción en el aula. Rosario: Homo Sapiens, 2005.

SANT OBIOLS, E.; CASAS ROS, M. T.; PAGÈS, J. Participar para aprender la democracia. Las representaciones sociales de jóvenes catalanes sobre la participación democrática. Uni-pluri/versidad Vol.11 No.2. Colombia, Medellín: Universidad de Antioquia, p. 1-25, 2011.

SANTISTEBAN, A. (2000) Aprender a enseñar el tiempo histórico: esquemas de conocimiento y perspectivas prácticas en el alumnado de formación inicial. En: PAGÈS I BLANCH, J.; ESTEPA GIMÉNEZ, J.; TRAVÉ GONZÁLEZ, G. (eds.) Modelos, Contenidos y Experiencias en la Formación del Profesorado de Ciencias Sociales. Barcelona: AUPDCS, 2000, p. 355-369. 
SANTISTEBAN, A. Una investigación sobre cómo se aprende a enseñar el tiempo histórico. Enseñanza de las Ciencias Sociales, Nº 6, p. 19-29, 2007.

SANTISTEBAN, A. Tiempo al tiempo en la escuela: una mirada transversal. Revista Perspectiva Escolar-Monografías. Enseñanza de las Ciencias Sociales, ${ }^{\circ}$ 1, p. 43-48, 2014.

SANTISTEBAN, A. Del tiempo histórico a la conciencia histórica: cambios en la enseñanza y el aprendizaje de la historia en los últimos 25 años. Revista Diálogo Andino $\mathrm{N}^{\mathrm{o}}$ 52, p. 87-99, 2017.

SANTISTEBAN, A.; ANGUERA, C. Las imágenes del futuro en los medios de comunicación y su influencia en la enseñanza de las ciencias sociales. En: DÍAZ MATARRANZ, J. J.; SANTISTEBAN FERNÁNDEZ, A.; CASCAJERO GARCÉS, A. (eds.). Medios de comunicación y pensamiento crítico. Nuevas formas de interacción social, Alcalá: Universidad de Alcalá - Servicio de Publicaciones / AUPDCS, 2013a, p. 253-267.

SANTISTEBAN, A.; ANGUERA, C. Education for the Future: estado de la cuestión de la investigación y de la innovación desde la enseñanza de las Ciencias Sociales. En: PAGÈS, J.; SANTISTEBAN, A (Eds.). Una mirada al pasado y un proyecto de futuro. Investigación e innovación en didáctica de las ciencias sociales. Vol. 1, Barcelona: Servei de Publicacions UAB / AUPDCS, 2013b, p. 659-667.

SANTISTEBAN, A.; ANGUERA CERAROLS, C. Formación de la conciencia histórica y educación para el futuro. Clio y Asociados (18-19), p. 249-267, 2014.

STAKE, R. Investigación con estudio de casos. Madrid: Ediciones Morata, 1999.

ZENOBI, V.; FLOUCH, A. Y GONZÁLEZ, V. Las representaciones sociales de la Geografía escolar: las voces de los estudiantes y los profesores. En Primeras Jornadas de la RedIEG, 2016. Bahía Blanca: Universidad Nacional del Sur, junio 2016, p. 1-17. 